\title{
Utilization of the internal transcribed spacer (ITS) DNA sequence to trace the geographical sources of Aquilaria malaccensis Lam. populations
}

\begin{abstract}
Overexploitation in search of its valuable non-wood fragrance product has put pressure on the survival of the endangered Aquilaria trees in the wild. In this study, the pattern of genetic variation among wild populations was measured to aid in designing useful strategies for in situ conservation. We sequenced the internal transcribed spacer (ITS) of the nuclear region of 19 wild Aquilaria malaccensis populations from different states in Peninsular Malaysia, and compared the sequence with the same species residing outside of Malaysia, mainly from the Assam region in India and Sumatra in Indonesia. This widely distributed species is found in the Indomalesian region and is a major source of agarwood. In addition, we included five wild Aquilaria hirta populations for semblance purposes. Intraspecific variations were not found within A. malaccensis and A. hirta populations in Peninsular Malaysia. Interestingly, Single Nucleotide polymorphisms (SNPs) were identified when comparing A. malaccensis from three geographical regions, with a total of 25 SNPs detected. We imply that geographical segregation is a contributing factor toward genetic variation in A. malaccensis. This is the first report on utilizing the ITS region for analysing genetic variation in $\mathrm{A}$. malaccensis of various geographical regions. The molecular information obtained in this study will serve as a useful reference in designing in situ programmes for this endangered species.
\end{abstract}

Keyword: Agarwood; Aquilaria hirta; Aquilaria malaccensis; Conservation; Genetic variation 